\title{
Téoros
}

Revue de recherche en tourisme

\section{Formation sur mesure : un outil qui bat la mesure!}

\section{Marie-Andrée Delisle}

Volume 11, numéro 1, mars 1992

La formation en tourisme : à la croisée des chemins

URI : https://id.erudit.org/iderudit/1078946ar

DOI : https://doi.org/10.7202/1078946ar

Aller au sommaire du numéro

Éditeur(s)

Université du Québec à Montréal

ISSN

0712-8657 (imprimé)

1923-2705 (numérique)

Découvrir la revue

Citer cet article

Delisle, M.-A. (1992). Formation sur mesure : un outil qui bat la mesure! Téoros, 11(1), 25-28. https://doi.org/10.7202/1078946ar d'utilisation que vous pouvez consulter en ligne.

https://apropos.erudit.org/fr/usagers/politique-dutilisation/ 


\section{Formation sur mesure: un outil qui bat la mesure!}

Marie-Andrée Delisle*

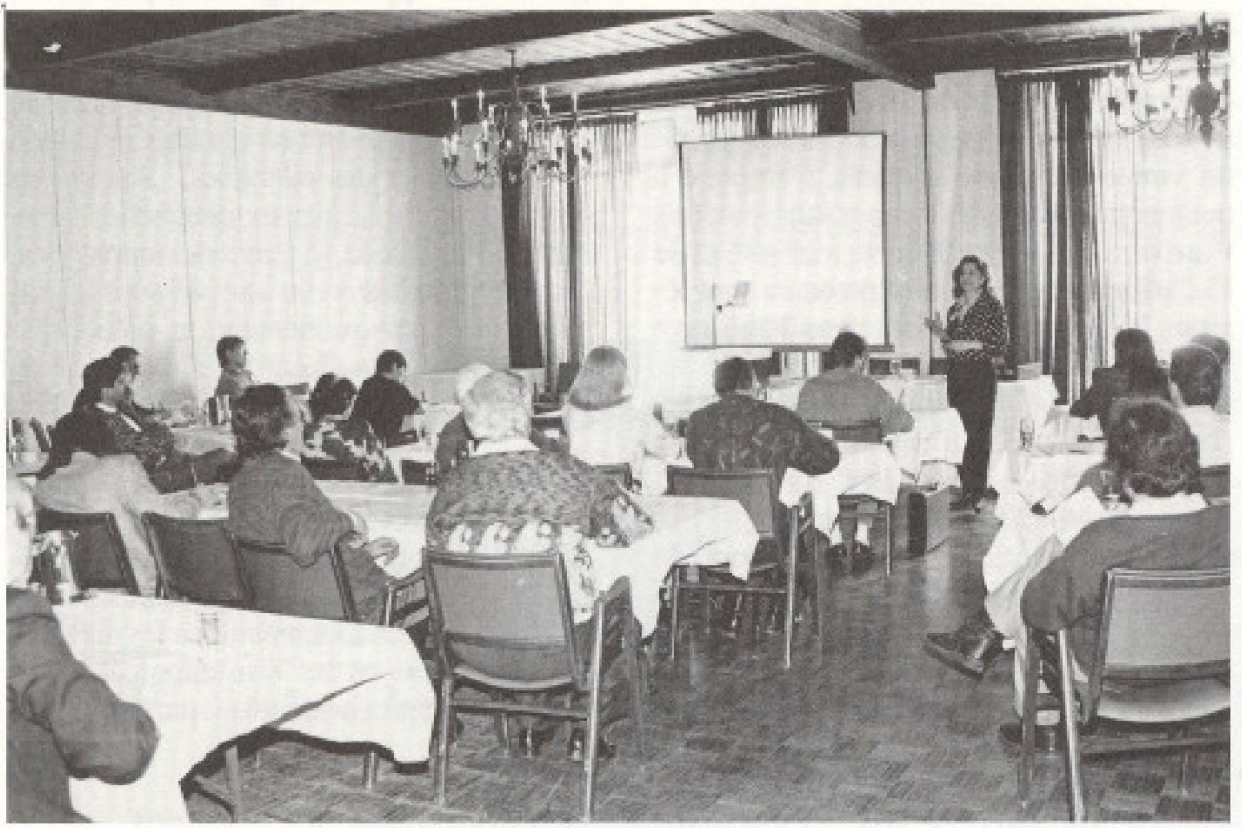

Srage de formaibn ser mesure anime por louteure a Baie Comear.

Sujet de l'heure, la formation semble suivre une mode. Pourtant, tout comme pour le concept de la qualité totale, nous en sommes encore à un niveau de sensibilisation en la matière. En fait, l'intérêt présent et pressant pour la formation sur mesure et la formation en entreprise fait suite à un changement beaucoup plus profond qu'il n'en laisse paraître.

Selon l'ICRT (Institut canadien de recherche sur le tourisme), sla formation et le perfectionnement professionnel figurent le plus souvent en tête de liste des priorités dans le domaine des ressources humaines chez les exploitants de tourisme en $1991 \%$ (1).

En transition entre une société industrielle et une société informationnelle, entre une démocratie de representation et une de participation, entre une hiérarchie et un réseau, le mouvement des relations humaines nous mène doucement vers la qualité de vie au travail, puis vers la qualité totale où le client,

Madame Marie-André Deliste est présidente de Marie-Andrée Delisle et Associés, fime-conseil en formation et en developperment organisationnel pour les PME en tourisme. l'employé et l'administration y trouvent leur compte!

Dans cette zone de transition, ce long tunnel qui doit mener vers un développement durable, les ressources humaines s"avèrent être les forces motrices de la survie, du maintien et du développement des organisations. $\mathrm{A}$ ce compte, la formation devient un important outil de gestion et de mobilisation.

En effet, la rapidité des processus, la compétitivité, l'exigence de qualité, la vitesse de production, la performanceet l'efficience sont autant de facteurs pouvant renforcer le fragile equilibre d'une entreprise, qui doit compter de plus en plus sur l'implication de son personnel. Pour demeurer en vie, $1^{*} \mathrm{en}-$ treprise d'aujourd 'hui doit pouvoir compter sur l'implication de son personnel. Pour ce faire, elle se doit de lui donner tous les outils nécessaires à la bonne marche des opếrations, par une formation adéquate, adaptée et rapidement utilisable; d'où la forte demande pour des programmes de formation sur mesure.

Quelque part aussi, la notion de gaspillage fait son chemin dans toutes les sphères.
L'écologie, la protection de l'environnement viennent déteindre sur nos façons de consommer et de gérer. Dans cet ordre d'idée, la formation surmesure se dessine comme Ctant un moyen efficaceet immédiai pour revitaliser les ressources humaines et pour motiver l'individu, lui permettant de mettre à profit son poteniiel, ses habiletes, ses aptitudes et son vécu de travailleur touten l'incitant à des notions d'excellence et de qualité.

\section{En quoi diffère la formation sur mesure?}

Définie comme étant un programme adapté à une entreprise, à une collectivite (une association, par exemple) ou àdes individus, la formation sur mesure traite de sujets ou de techniques specifiques (produits, services, technologie, etc.). Elle prend en considération le secteur d'industrie auquel elle s'adresse. Si l'on parle de formation en service à la clientèle en milieu hôtelier, on intégrera dans le contenu l'environnement de ce type d'établissement, en s'assurant de comprendre les particularites du fonctionnement interne, de l'ancienneté du personnel, de l'historique de l'entreprise, des politiques et procédures, du profil de clientèle, et de toute autre caractéristique propre à cet hôtel.

Dans le même ordre d'idé, le système de réservations Apollo d'Air Canada ou le Sabre d'American Airlines sera enseigné selon les besoins de l'agent de voyages et de son environnement, dans un langage qui lui est familier et selon différentes étapes d'application propres aux clientèles d'affaires ou d'agrément. Un voyagiste pourra demander une formation sur la gestion du stress et des situations difficiles pour ses représentants à destination; la formation sera alors adaptée a des situations rélles en rapport directavec le vécu de l’employé et en réponse à des difficultés.

Une centrale de réservations demandera une formation sur les techniques téléphoniques, une autre sur les techniques de vente; encore ici, l'étude du produit, de son environnement de marche, de la problématique du produit, de ses caractéristiques et avantages, du profil de clientele ainsi que des politiques de l'en- 
treprise devront être pris en considération afin de mouler la technique à l'entreprise, et non, comme on le voit souvent, de mouler l'entreprise à la technique ou à la théorie!

On pourrait également définir la formation sur mesure par la nécessité qu'elle soustend celle de bien identifier les objectifs du groupe et/ou de l'employeur et de recueillir les nombreux indices nécessaires a l'adaptation du contenu au vécu des participants.

\section{Où s'inscrit la formation sur mesure?}

Parmi les grands schémas de formation dans son sens le plus large et le plus générique, la formation sur mesure vise plusieurs objectifs: elle sert parfois de rattrapage aux entreprises qui ont tardéà outiller leur personnel avec de nouvelles technologies ou de les familiariser avec les plus récentes approches marketing. A cecompte, lesrésultats doivent être immédiats, rapides, concluants.

La formation sur mesure vise des objectifs a court terme, tels le maintien ou l'adaptation à de nouveaux systèmes informatiques comme apprendre un traitement de texte, ou administratifs comme intégrer une nouvelle méthode de gestion. Elle sert des objectifs a moyen et à long terme qui viseront à structurer des standards de performance, que ce soit en service au client ou en qualité totale, et d'implanter des moyens d'action et de suivi.

A ces objectifs devra automatiquement $s$ ajouter une recherche constante d'amélioration des produits, des services et du rendement humain si l'entreprise entend demeurer dans la course et continuer à croître dans les années 2000.

Sur mesure, la formation sert ainsi à assurer le développement de l'employé et de l'entreprise qui mise sur ses compétences. Comme on le voit, le terme sur mesure n"implique pas nécessairement qu'une formation soit de courte durée.

\section{De plus en plus en demande}

Il ne faut pas se le cacher, la formation sur mesure trouve davantage preneur en temps de récession, alors que les entreprises ont davantage de tempsetde disponibilitéd'esprit pour y réfléchir. Cependant, de plus en plus, les organisations prennent des initiatives d'implantation de qualitế qui nécessitent l'usage de séminaires et d'ateliers de forma- tion pointue et sur mesure. En effet, si le client devient le vrai focus d'une entreprise qui cherche à se démarquer, cette dernière se devra d'implanter des programmes de formation qui lui sont propres, d'où l' accroissement de la demande pour du sur mesure.

Mais d'où vient la demande? Malheureusement, elle vient souvent d'un malaise, d'un cul-de-sac dans lequel peut se retrouver une entreprise quand elle se voit déraper. L'entreprise identifie certains besoins, entreprend un virage-focus sur le client, s'emploie à maintenir le moral des troupes sans toutefois y arriver. Dans le secteur du tourisme et de $1^{t}$ hôtellerie, les petits entrepreneurs sont légion. Ils feront appel à des consultants ou à des spécialistes pour les aider à contoumer ces problèmes en outillant leur personnel pour faire face aux changements dans leurs façons de procéder et de gérer. Bien souvent les employeurs, tout comme leurs employes, ne sont pas en position de suivre des cours de niveau collégial ou universitaire: ou bien ils ne possedent pas les pré-requis, ou bien ils n'ont pas la motivation pour suivre des cours généraux qui pourraient leur servir un jour, ou encore ils sont pressés d'arriver à des résultats très souvent décisifs par rapportà la survie de leur entreprise ou de leur travail. Ils n'ont ni le temps ni la disponibilité pour s'engager dans des formations de longue durée: ils sont déjà dans le bain! Leurs connaissances générales ne sontpas toujours solides, mais ce ne sont pas nécessairement que les connaissances qui font de bons employes. Le fait d'avoir acquis une formation de base leur permettra certainement une ouverture d'esprit, un intérêt etune créativité différente face aux défis des années 1990, mais leur attitude et leur personnalité pèseront lourd dans la balance. Comme on dit souvent, on embauche une personne pour ses compétences... et on la congédie pour son attitude!

Par ailleurs, l'enseignement dit régulier ne colle pas toujours à la réalité. Malgré que l'on ne puisse généraliser, loin de là, certains professeurs tant au collégial qu'à l'université n'ont pas revu leurs notes depuis belle lurette et demeurent confortablement assis sur des préparations de cours poussiéreuses. Plusieurs n'ont aucune expérience de terrain et parlent de généralités qui tuent l'intérêt dans l'oeuf. En outre, des professeurs à plein temps risquent de se retrouver coupes de la réalité de l'industrie, sans possibilité pour eux de se recycler ou d'effectuer des stages en entreprise.
En comparaison, la majorité des formateurs affectés à la formation sur mesure et issus des milieux scolaires, collégiaux et universitaires sont généralement compétents dans leurs spécialités et travaillent conjointement avec les entreprises à développer des standards de qualité et d'efficacité par le biais de programmes d'apprentissage ou de perfectionnement. Aussi, leur faut-il se documenter continuellement, effectuer des recherches et travailler sur le terrain pour se maintenir au rythme extrêmement rapide de l'évolution des marches et des clientèles. Soulignons entre autres les nouveaux débouchés informatiques en fait de systèmes de réservations, l'intérêt grandissant du touriste pour la protection de l'environnement, la préoccupation du ratio qualite/prix, l'exigence de l'accueil et du service aux clients, etc.

A ce sujet, on note souvent que les méthodes et théories préconisées dans les cours magistraux de formation collégiale et universitaire ne répondent plus aux tendances du marché: entre le moment où on les digère et le moment où on les institutionnalise, ces notionss'avèrent inadéquates par rapportà la waie vie!

\section{Des demandes qui viennent de tous les secteurs}

Les fournisseurs de services de formation sur mesure reçoivent des demandes de tous les secteurs de l'industrie touristique. En hôtellerie et en restauration: gestion du personnel, contrôle des coutts, gestion des horaires, service aux tables; en ventes et marketing: approche teléphonique, techniques de vente pour agents de voyages, pour agents de réservations, pour vendeurs de produits touristiques;en informatique: gestion de dossiers, gestion comptable, réservations, en notions de base pour des plans marketing de petites entreprises, en ćlaboration de forfaits pour des propriétaires d'attraits touristiques ou des organisateurs d'événements, en sensibilisation aux différents réseaux de distribution, en motivation du personnel, consolidation d'équipe et supervision, en sensibilisation a l'accueil et au service à la clientèle.

Des offices de tourisme, des associations touristiques, des stations de ski, des agences de voyages, des voyagistes, des hôtels, des transporteurs, des organisateurs d'événements font appel à des consultants en formation pour des besoins spécifiques. Certaines formations regroupent des entrepreneurs, comme dans le cas des formations de thèmes 
sur mesure que met de l'avant le ministère du Tourisme pour les intervenants de l' industrie, comme la formation effectuée aupres des vendeurs québécois pour préparer efficacement leur participation à des bourses touristiques. D'autres visent l'ensemble des employés affectés aux divers secteurs d'une entreprise, comme tout le personnel d'un centre de ski.

Malgré l'établissement d'objectifs spécifiques, l'expérience démontreque,peuimporte leur åge et leur ancienneté, le niveau de motivation et d'implication des employés s'accroit des le début de l'intervention. En effet, ces demiers sont heureux de recevoir de nouveaux outils qui visent à faciliter leur travail. De plus, le fait d' intégrer leur vécu permet de coller à leur réalité et de répondre adéquatement aux difficultés et besoins exprimés. Le défi demeurera cependant dans le camp de l'administration qui se devra de maintenir le degré de motivation en instaurant des programmes continus de perfectionnement.

\section{Différentes formules}

- Lesétablissementsd'enseignement, tels que l'École Hôtelière des Laurentides, le CÉGEP de Ste-Foy, le Collège Edouard-Montpetit, l'UQAM, I'Université McGill, les HEC, offrent une panoplie de cours de formation sur mesure visant à combler les besoins en gestion et en operations des différents types d'entreprises touristiques. Ces formations peuvent durer de quelques heures à quelques semaines.

- De nombreuses firmes de consultants proposent des formations diverses en gestion dont les sujets peuvent s'adapter aux besoins de tout gestionnaire de l'industrie; on n'a qu'à feuilleter le cahier spécial Formation et perfectionnement du journal Les Affaires ${ }^{(2)}$ qui paraîtdeux foisl $l^{t}$ an, pour se rendre compte de l'eventail de services de cet ordre. D'autres encore se regroupent pour présenter des journées de formation faisant appel à une clientè̀le générale.

- Viennent ensuite les formateurs specialisés qui se font plutôt rares en tourisme et en hôtellerie; ces derniers proposent des outils pratiques et adaptés aux réalités de l'industrie, tant dans le langage que dans la problématique.
- Plusieurs firmes de formation sont enregistrées auprès de la Commission de formation professionnelle $(C F P)$ afin de faire profiter leurs clients des mesures fiscales de remboursement de crédit d'impôt. Encore ici, les petites entreprises de consultants se frottent aux départements de formation de moyennes et grandes entreprises, dont certaineschaînes d'hôtels. Desagences telles Voyages Bel-Air et Voyages Sears possedent leurs propres directeurs de formation qui embauchent des formateurs spécialisés selon leurs besoins. Certaines associations telles $\mathrm{l}^{\prime}$ Institut canadien des conseillers en voyages (CITC) proposent une fois l'an un colloque de deux jours sur le marché des comptes corporatifs, avec des ateliers, des conférences et des débats sur les sujets de l'heure.

- Les séminaires jouentégalement un rôle de ressourcement pour certains gestionnaires. Dans le cas d'entreprises établiesetrelativement bien structurées, l'on préférera plutôt participer à des colloques de ressourcement personnel et professionnel où l'on pourra étendre son réseau de connaissances et échanger sur des préoccupations de gestion.

Dimension clientèle, une association québécoise de professionnels et professionnelles du service a la clientele propose pour sa part des petits-déjeuners causerie où des conférenciers discutent de cas, dans un but d'echange et de perfectionnement. Enfin, des journées-conférence comme celle sur les Perspectives touristiques du Québec - 1992, organisces par l'ICRT et l'OCTGM (Office des congrès et du tourisme du Grand Montréal), permettent aux intervenants de prendrele pouls du marché et de s'enligner sur les nouvelles orientations que dicte l'économie et les tendances de l'an 2000 .

\section{De l'aide financière pour réduire les coûts de formation}

Plusieurs types de subventions peuvent aider les entreprises à intégrer un plan de formation dans la gestion de leur personnel et de leurs opérations. La meilleure porte à laquelle frapper, tant pour être orienté que pour être informé, demeure celle de la Commission de formationprofessionnelle(CFP) quiregroupe tous les programmes d'aide à la formation et offre de la consultation en stratégie de formation. De nombreuses formules sont proposées aux petites et moyennes entreprises,

\begin{tabular}{|ll}
\hline C.F.P. & $\begin{array}{l}\text { Commission de formation } \\
\text { professionnelle } \\
\text { (bureaux régionaux - pages } \\
\text { blanches de l'annuaire télé- } \\
\text { phonique) }\end{array}$ \\
P.S.F.E. & $\begin{array}{l}\text { Programme de soutien à la } \\
\text { formation en entreprise }\end{array}$ \\
P.D.R.H. & $\begin{array}{l}\text { Programme de développe- } \\
\text { mentdesressourceshumaines }\end{array}$ \\
F.M.E. & $\begin{array}{l}\text { Formation sur mesure en } \\
\text { établissement }\end{array}$ \\
\hline
\end{tabular}

allant du programme de soutien à la formation en entreprise (PSFE), à la formation sur mesureen établissement(FME), aurecyclage etperfectionnement professionnel de lamaind'oeuvre et au crédit d'impôt à la formation (achat d'un plan de développement des ressources humaines; frais de formation et salaires admissibles).

Certains programmes sont subventionnes a $100 \%$, selon des conditions précises d'application, soit entre autres un nombre d'heures, un nombre de participants et un contenu decours réglementé. Certains autres proposent, comme c'est le cas du credit d' impôt, jusqu'à $40 \%$ de remboursement des coûts de formation avec un nombre infime de contraintes.

Une entreprise voyagiste de Montréal ainsi qu'un hôtelier de la ville de Québec se prévalent présentement d'une complémentarité de programmes qui leur permettent de financer un PDRH à $75 \%$ (jusqu'à concurrence de 10000 \$) dans le cadre d'un PSFE. Le plan de developpement de ressources humaines, communément appele PDRH, est une analyse de tous les besoins de formation d'une compagnie, au niveau informatique, comptable, administratif, opérationnel et humain. Ce plan est effectué par un consultant conjointement choisi par l'entreprise et laCFP, dans le cadre du PSFE. Le consultant met généralement trois mois à effectuer son analyse qu'il présente à l'employeur sous forme de rapport. Ce diagnostic est une des façons les plus efficacesd'avoirl'heure juste a tous les niveaux de son organisation. Une fois le plan déposéavec les recommandations qui s'imposent en formation, l'employeur pourra ensuite amorcer un programme selon les priorités qui ressortent du rapport, et échelonner sur trois ans les activités de formation qu'il entend compléter. Encore ici, la 
CFP pourra lui faire valoir d'autres mesures fiscales qui diminueront considérablement ses coûts d'implantation. D'autre part, la Société de développement industriel (SDI) propose des programmes d'aide à la relance et à l'investissement (prêts minimum de 20 $000 \$$ ), pour lesquels les grandes entreprises touristiques sont admissibles.

Avec son nouveau programme de crédits d'impôt à la formation, le gouvernement incite davantage l'entreprise à investir dans un budget de formation annuel. Selon les statistiques, le Québec ne dépense que $0,3 \%$ de la masse salariale en formation, comparativementavec $0,5 \%$ au Canada, $1,4 \%$ aux Etats-Unis et $4 \%$ au Japon ${ }^{(3)}$.

\section{Que dire de la qualité de la formation sur mesure?}

Au départ, l'individu ou l'entreprise doit analyser ses besoins a l'aide de sondages ou avec l'aide d'un consultant. Plus l'analyse serajuste, et plus les objectifs seront détaillés, plus précis seront les résultats. Par ailleurs, l'on serend souventcomptequ' uneentreprise fait appel à des consultants pour un besoin précis, alors qu'en réalité ce besoin apparent cache un autre type d'intervention à entreprendre: consolidation d'équipe, réorganisation des fonctions, refonte de l'organigramme, redistribution des tâches, motivation du personnel, engagement de l'administration envers ses employés.

Bien qu'il soit très difficile d'évaluer quantitativement les effets des programmes de formation, soulignons que la qualité des rapports, la qualité du service, le moral et la satisfaction du personnel s'ameliorent sensiblement, alors que le taux de roulement faiblit considérablement.

Ces résultats ne vont pas sans avoir d'effets bénéfiques sur la productivité et le climat de travail!

Le choix du consultant aura Également une incidence sur le résultat. Sa capacité de transmettre des notions de savoir-faire aux adultes apprenants, son expertise dans le domaine et ses références sont définitivement des atouts.

Mais l'important, c'est de ne pas prendre la formation sur mesure comme un bouchetrou ou du fast food. La formation doit demeurer une préoccupation constante d'amélioration du personnel et des procédés.
Un petit programme de quelques heures ne feraquesensibiliser l'individuà une nouvelle approche, mais les résultats s'effriteront rapidement.

La problématique d'ensemble et la voie future de la formation sur mesure sont directement liés a la prise de conscience des entreprises du besoin de faire corps avec leur personnel. La mission d'entreprise, l'intégration du concept de qualité et de service ne peuvent se faire sans la participation des employés. La revitalisation des ressources humaines, par la motivation, la considération et la reconnaissance passent sans contredit par des programmes de formation continus et sur mesure. Sinon, comment demeurer dans la course et s"adapter aux changements de plus en plus rapides du marché local et mondial? Faut-il repenser le rôle de la formation régulière? Oui et non. La refonte du programme 414 de techniques touristiques au niveau collégial propose maintenant une methode de formation par compétence, ce qui nous rapproche davantage du vécu quotidien et des habiletés d'une personne à performer habilement et avec entrain. Par ailleurs, les méthodes changent, les besoins, les habitudes d'achat, les priorités bougent. Que vaut maintenant un diplôme vieux de dix ans, sinon la gymnastique mentale, la structure de pensée, les associations d'idées, les méthodes de recherche qui auront aiguisé le discernement et ouvert l'esprit au monde?

Embauchera-t-on pour autant des personnes non qualifiées? Pour sûr que non. En autant que les diplômés acquerront, en même temps que leurs connaissances, des qualités de travailleurs: dynamiques, disciplinés, serviables et soucieux du travail bien fait.

La formation est devenue un elément constant dans la vie de tout travailleur, qu'il soit professionnel ou opérationnel. Qu'on le veuille ou non, ce n'est pas de sitôt qu'on quittera les bancs d'école!

\section{NOTES ET RÉFÉRENCES}

(1) Kimberley Bachmann, La fomation: pourquoi?, Le tourisme en perspectlve, Bulletin de I'Institut canadien de recherche sur le tourisme, vol. 4, no 2, hiver 1991.

(2) Cahier Famation et Perfectionnement, Le Journal Les Affaires, edition du 11 janvier 1992.

(3) Jean-Benoit Nadeau, A l'école... buissonnière, Revue PME, juillet-août 1991. 